\title{
A Simple Sensor Model for THUNDER Actuators
}

Joel F. Campbell and Robert G. Bryant

NASA Langley Research Center, MS 488

Hampton, VA 23681

\begin{abstract}
A quasi-static (low frequency) model is developed for THUNDER actuators configured as displacement sensors based on a simple Raleigh-Ritz technique. This model is used to calculate charge as a function of displacement. Using this and the calculated capacitance, voltage vs. displacement and voltage vs. electrical load curves are generated and compared with measurements. It is shown this model gives acceptable results and is useful for determining rough estimates of sensor output for various loads, laminate configurations and thicknesses.
\end{abstract}




\section{Introduction}

Thunder actuators are devices constructed from an isotropic laminate of aluminum, LaRC-SI adhesive, an electroded Lead Zirconate Titanate (PZT) piezoelectric ceramic wafer and a metal backing of either steel or brass. The top aluminum layer is used to increase the durability of the laminate and is sometimes omitted. The LaRC-SI adhesive is a 1-2 mil thick solid thermoplastic film, and the aluminum layer is indented to provide electrical conductivity to the wafer after the actuator is pressure bonded above $250^{\circ} \mathrm{C}$. This hot-melt bonding process induces a mechanical prestress in the THUNDER laminate that results a bow shape actuator. This curve is the result of the mismatch in the Coefficient of Thermal Expansion (CTE), of the PZT and the metallic prestress layer, as the bonded laminate cools to room temperature. If simply supported or arranged in a clamshell arrangement the THUNDER wafers produce a linear motion proportional to the externally applied voltage. These actuators may also be used as sensors due to the intrinsic piezoelectric effect. Since the construction of the THUNDER wafers can be varied to a great extent by the selection of materials, processing conditions and geometry, it became apparent that computational and analytical models were needed to guide the construction of THUNDER wafers to provide practical solutions to engineering problems.

Analytic THUNDER models span more than a decade. The very first was developed by Alan R. D. Curtis in December, 1997 who was hired as a NASA contractor through BBN Technologies[1]. The BBN model was a very simple linear model that assumed only 3 layers, but was a good first effort. In that same timeframe a modeling 
group at Langley was setup headed by Bill Hunter who was chosen to coordinate the modeling efforts of various individuals involved in modeling THUNDER. This group consisted of Joel Campbell, Barmac Taleghani [2], and Jill Marlow. Taleghani and Marlow were working on finite element models based on NASTRAN and Campbell was working on an analytic model.

In December, 1997 Hunter introduced Campbell to M. W. Hyer's work in composite laminates [3-5]. Campbell reformulated Hyer's work for isotropic media and piezoelectric expansion, and thus was able to apply Hyer's composite modeling to THUNDER. Piezoelectric actuation is analogous to thermal expansion so modifying those models for piezoelectric layers was not difficult. By January, 1998 Campbell had a fully functional model and by February 1998 he submitted two papers to Smart Materials and Structures for publication, which were accepted in July on condition of submitting a minor revision. The first of these papers detailed the basic model for rectangular actuators and the second dealt with a method for predicting blocked force. By March he had a model for circular THUNDER actuators and he submitted a patent disclosure, LAR15827, based on all 3 papers in April, 1998. He was asked by the NASA patent office not to publish those results until an intellectual property judgment could be made, so the revision was withheld. Due to a serious backlog this process took over 5 years and by then the work was no longer current. Unfortunately, the decision was made not to pursue a patent, but disclosed the content to Virginia Power, now Dominion Energy, under their THUNDER license agreement. These 3 papers have since been republished as NASA TM's [6-8] for historical purposes.

During this timeframe Hyer was collaborating with a separate NASA group on THUNDER. He independently developed an analytical model of his own that he submitted 
to Smart Materials and Structures in April of 1998 [9], two months after Campbell's submission to the same journal. This model was very similar to Campbell's first paper [6] with the following exceptions. First, Hyer formulated his energy integral in terms of forces and moments whereas Campbell followed Hyer's earlier formulation in terms of stresses and strains. Although this may look different superficially, the formulation is equivalent. Another difference is Campbell's formulation assumed purely isotropic layers whereas Hyer's model was more general, similar to Hyer's composites work. Campbell also takes into account external loading in his model for blocked force. Hyer then followed this up with a model for circular actuators in 2002 and 2003 [10-11]. This was a more sophisticated model than Campbell's circular actuator model from 1998 in that it used higher order polynomials. Hyer's group has since perfected his rectangular actuator model to include higher order polynomials and 21 independent coefficients, compared to 4 in the earlier model [12], which has been verified experimentally by Mossi et al[13]. Other modeling efforts by others include what appears to be a more sophisticated version of the BBN model [14], and some very interesting work modeling hysteresis [15].

There has been much less work modeling THUNDER as a sensor, with only two published papers using finite element analysis [16-17]. Campbell, however, developed a model for THUNDER as a sensor back in 1998 complete with software and examples. Unfortunately, due to other commitments, this work was abandoned before the report was completely finished. That report was an attempt to model experimental data that had been collected by a student a few moths before, and to realize THUNDER actuators as potential power sources for energy harvesting. This paper is based on that unpublished report. No analytic model for THUNDER actuators as sensors has been published in the past decade. This is surprising considering the interest in using THUNDER actuators as 
sensors and energy harvesters. This paper represents a first contribution to that effort, which is useful to those who neither have nor wish to use finite element software.

\section{The Basic Model}

The Von Karman approximation assumes large displacement and small strain. This suggests including second order terms in $\mathrm{w}$ in addition to the linear terms for $\mathrm{u}, \mathrm{v}$ and w. Expressing the strain to include these terms gives[18],

$$
\begin{aligned}
& \varepsilon_{x} \approx \frac{\partial u}{\partial x}+\frac{1}{2}\left(\frac{\partial w}{\partial x}\right)^{2}, \\
& \varepsilon_{y} \approx \frac{\partial v}{\partial y}+\frac{1}{2}\left(\frac{\partial w}{\partial y}\right)^{2}, \\
& \gamma_{x y} \approx \frac{\partial u}{\partial y}+\frac{\partial v}{\partial x}+\frac{\partial w}{\partial x} \frac{\partial w}{\partial y}
\end{aligned}
$$

By using the relation,

$$
\begin{aligned}
& u=u_{0}-z \frac{\partial w_{0}}{\partial x} \\
& v=v_{0}-z \frac{\partial w_{0}}{\partial y}
\end{aligned}
$$

we find, 


$$
\begin{aligned}
& \varepsilon_{x} \approx \frac{\partial u_{0}}{\partial x}+\frac{1}{2}\left(\frac{\partial w_{0}}{\partial x}\right)^{2}-z \frac{\partial^{2} w_{0}}{\partial x^{2}}, \\
& \varepsilon_{y} \approx \frac{\partial v_{0}}{\partial y}+\frac{1}{2}\left(\frac{\partial w_{0}}{\partial y}\right)^{2}-z \frac{\partial^{2} w_{0}}{\partial y^{2}}, \\
& \gamma_{x y} \approx \frac{\partial u_{0}}{\partial y}+\frac{\partial v_{0}}{\partial x}+\frac{\partial w_{0}}{\partial x} \frac{\partial w_{0}}{\partial y}-2 z \frac{\partial^{2} w_{0}}{\partial x \partial y}
\end{aligned}
$$

where $\mathrm{u}_{0}, \mathrm{v}_{0}$, and $\mathrm{w}_{0}$ are the middle thickness displacements in the $\mathrm{x}, \mathrm{y}$, and $\mathrm{w}$ direction. See Figure 1.

The total energy takes the form

$$
U=\iiint\left(d U_{0}-d W_{T}-d W_{A}\right)-\iint w p(x, y) d x d y
$$

where $\mathrm{dU}_{0}$ is the stored elastic energy volume density and $\mathrm{dWT}_{\mathrm{T}}$ is the temperature contribution. Excluding the pressure term, this follows that used by others $[3-5,19]$. The last integral is the work done against the external pressure, p. The elastic energy portion for the $k^{\prime}$ th layer can be found with the help of [20],

$$
\begin{aligned}
& \left(d U_{0}\right)_{k}=\frac{1}{2}\left(\sigma_{x} \varepsilon_{x}+\sigma_{y} \varepsilon_{y}+\tau_{x y} \gamma_{x y}\right)_{k} d x d y d z= \\
& \left(\frac{1}{2} Q_{1} \varepsilon_{x}^{2}+Q_{2} \varepsilon_{x} \varepsilon_{y}+\frac{1}{2} Q_{1} \varepsilon_{y}^{2}+\frac{1}{2} Q_{3} \gamma_{x y}^{2}\right)_{k} d x d y d z
\end{aligned}
$$

For the thermal portion we have 


$$
\begin{aligned}
& \left(d W_{T}\right)_{k}=\left(\sigma_{x}^{T} \varepsilon_{x}+\sigma_{y}^{T} \varepsilon_{y}\right)_{k} d x d y d z= \\
& \left(\left(Q_{1}+Q_{2}\right) \alpha \Delta T \varepsilon_{x}+\left(Q_{1}+Q_{2}\right) \alpha \Delta T \varepsilon_{y}\right)_{k} d x d y d z
\end{aligned}
$$

For the actuation portion we have

$$
\begin{aligned}
& \left(d W_{A}\right)_{j}=\left(\sigma_{x}^{A} \varepsilon_{x}+\sigma_{y}^{A} \varepsilon_{y}\right)_{j} d x d y d z= \\
& \left(Q_{1}+Q_{2}\right)_{\mathrm{j}} d_{31} \frac{\mathrm{V}}{\mathrm{t}} \varepsilon_{x}+\left(Q_{1}+Q_{2}\right)_{\mathrm{j}} d_{31} \frac{\mathrm{V}}{\mathrm{t}} \varepsilon_{y} d x d y d z
\end{aligned}
$$

Actuation is assumed to occur on the $j$ 'th layer and $Q$ is the reduced stiffness with $\mathrm{Q} 11=\mathrm{Q} 22=\mathrm{Q} 1$ and $\mathrm{Q} 12=\mathrm{Q} 21=\mathrm{Q} 2$. Now that we have the general form for the energy integral we may try a Raleigh-Ritz type solution. The method involves making a guess for the solution. This guess should look qualitatively like the expected solution yet be flexible enough to allow for adjustment. This adjustment comes in the form of changing the guess based on a finite number of parameters, which the guess contains. The parameters are adjusted in such a way as to minimize the total energy of the system. In this situation it would be advantageous to choose a guess that is close enough to the classical lamination result that in the linear limit (small scaling) the solution approaches the classical lamination result. Such a guess takes the form

$$
w^{0}=w_{0}^{0}+\frac{1}{2}\left(a x^{2}+b y^{2}\right)
$$


Classical lamination theory assumes no shearing strain between layers for thermal expansion. If we make the same assumptions here we find $u^{0}$ and $v^{0}$ must take the form

$$
\begin{aligned}
& u^{0}=c x-\frac{1}{6} a^{2} x^{3}-\frac{1}{4} a b x y^{2}, \\
& u^{0}=d y-\frac{1}{6} b^{2} y^{3}-\frac{1}{4} a b x^{2} y
\end{aligned}
$$

The ansatz represented by Equation 8 and 9 has been used successfully by others in the treatment of laminates and actuators [3-5, 6-8]. Equations 3,8 and 9 give

$$
\begin{aligned}
& \varepsilon_{x}=c-\frac{1}{4} a \mathrm{~b} \mathrm{y}^{2}-a \mathrm{z}, \\
& \varepsilon_{y}=d-\frac{1}{4} a \mathrm{~b} \mathrm{x}^{2}-b \mathrm{z}, \\
& \varepsilon_{x y}=0
\end{aligned}
$$

If the length in the $x$ direction is $L_{x}$ and the length in the $y$ is $L_{y}$ then, we may approximate $\mathrm{w}_{0}^{0}$ by,

$$
w_{0}^{0}=-\frac{1}{8}\left(a \mathrm{~L}_{\mathrm{x}}^{2}+b \mathrm{~L}_{\mathrm{y}}^{2}\right)
$$

This assumes the device is simply supported. For $\mathrm{p}(\mathrm{x}, \mathrm{y})$ we choose a point load of,

$$
p(x, y)=F \delta(x) \delta(y)
$$

Other choices are possible such as a constant distributed pressure. However, a point load is closest to the type of load used with the rectangular devices. The solution is 
obtained by minimizing the total energy with respect to a, b, c and d. The result of the minimization is,

$$
\begin{aligned}
& D_{1} a+K_{8} b-B_{1} c-B_{2} \mathrm{~d}+\mathrm{K}_{1} \mathrm{~b}^{2}+K_{6} \mathrm{ab}-\mathrm{K}_{4} \mathrm{bc}-\mathrm{K}_{3} \mathrm{bd}+ \\
& \mathrm{K}_{7} \mathrm{ab}^{2}+M^{\prime}-\frac{1}{8}\left(\frac{L_{x}}{L_{y}}\right) F=0, \\
& K_{8} \mathrm{a}+\mathrm{D}_{1} \mathrm{~b}-\mathrm{B}_{2} c-B_{1} d+K_{2} \mathrm{a}^{2}+K_{5} \mathrm{ab}-\mathrm{K}_{4} \mathrm{ac}-\mathrm{K}_{3} \mathrm{ad}+ \\
& \mathrm{K}_{7} a^{2} b+M^{\prime}-\frac{1}{8}\left(\frac{L_{x}}{L_{y}}\right) F=0, \\
& -B_{1} a-B_{2} \mathrm{~b}+\mathrm{A}_{1} \mathrm{c}+\mathrm{A}_{2} \mathrm{~d}-\mathrm{K}_{4} \mathrm{ab}-\mathrm{N}^{\prime}=0, \\
& -B_{2} a-B_{1} \mathrm{~b}+\mathrm{A}_{2} \mathrm{c}+\mathrm{A}_{1} \mathrm{~d}-\mathrm{K}_{3} \mathrm{a} \mathrm{b}-\mathrm{N}^{\prime}=0
\end{aligned}
$$

where,

$$
\begin{aligned}
& K_{1}=\frac{1}{48}\left(B_{1} L_{x}^{2}+B_{2} L_{y}^{2}\right), \\
& K_{2}=\frac{1}{48}\left(B_{2} L_{x}^{2}+B_{1} L_{y}^{2}\right), \\
& K_{3}=\frac{1}{48}\left(A_{1} L_{x}^{2}+A_{2} L_{y}^{2}\right), \\
& K_{4}=\frac{1}{48}\left(A_{2} L_{x}^{2}+A_{1} L_{y}^{2}\right), \\
& K_{5}=\frac{1}{24}\left(B_{1} L_{x}^{2}+B_{2} L_{y}^{2}\right), \\
& K_{6}=\frac{1}{24}\left(B_{2} L_{x}^{2}+B_{1} L_{y}^{2}\right), \\
& K_{7}=\frac{1}{1152} A_{2} L_{x}^{2} L_{y}^{2}+\frac{1}{1152} A_{1}\left(L_{x}^{4}+L_{y}^{4}\right), \\
& K_{8}=D_{2}+\frac{N^{\prime}}{48}\left(L_{x}^{2}+L_{y}^{2}\right)
\end{aligned}
$$


The A's, B's and D's have the usual meanings of extensional, coupling and bending stiffness. The force and moment per unit width, $\mathrm{N}^{\prime}$ and $\mathrm{M}^{\prime}$, are given by,

$$
\begin{aligned}
& N^{\prime}=\Delta T \sum_{k=1}^{N} \alpha_{k}\left(Q_{1}+Q_{2}\right)_{k}\left(z_{k}-z_{k-1}\right), \\
& M^{\prime}=\frac{\Delta T}{2} \sum_{k=1}^{N} \alpha_{k}\left(Q_{1}+Q_{2}\right)_{k}\left(z_{k}^{2}-z_{k-1}^{2}\right)
\end{aligned}
$$

The $z^{\prime}$ s are measured with respect to the middle surface and $z_{0}$ is the top surface. The lengths $L_{X}$ and $L_{y}$ are the length and width of the actuator. It is a simple matter to show that the solution in the linear limit (small scaling $-\mathrm{L}_{\mathrm{x}}=\mathrm{L}_{\mathrm{y}}=0$ ) matches the classical lamination result if we take $a=-\kappa_{x}, b=-\kappa_{y}, c=\varepsilon_{x}$ and $d=\varepsilon_{y}$ and $F=0$.

Unlike the classical lamination result, the non-linear analysis predicts unequal curvatures that depend on the magnitude of the scaling and the aspect ratio. It also predicts multiple possible solutions. The nature of these solutions are dome-like solutions which approach cylinders in the limit of large scaling. Under load, saddles are also possible. Typically, there will be one or two stable solutions and possibly a third unstable solution depending on the size and material properties. There are also unphysical solutions in terms of complex numbers. 


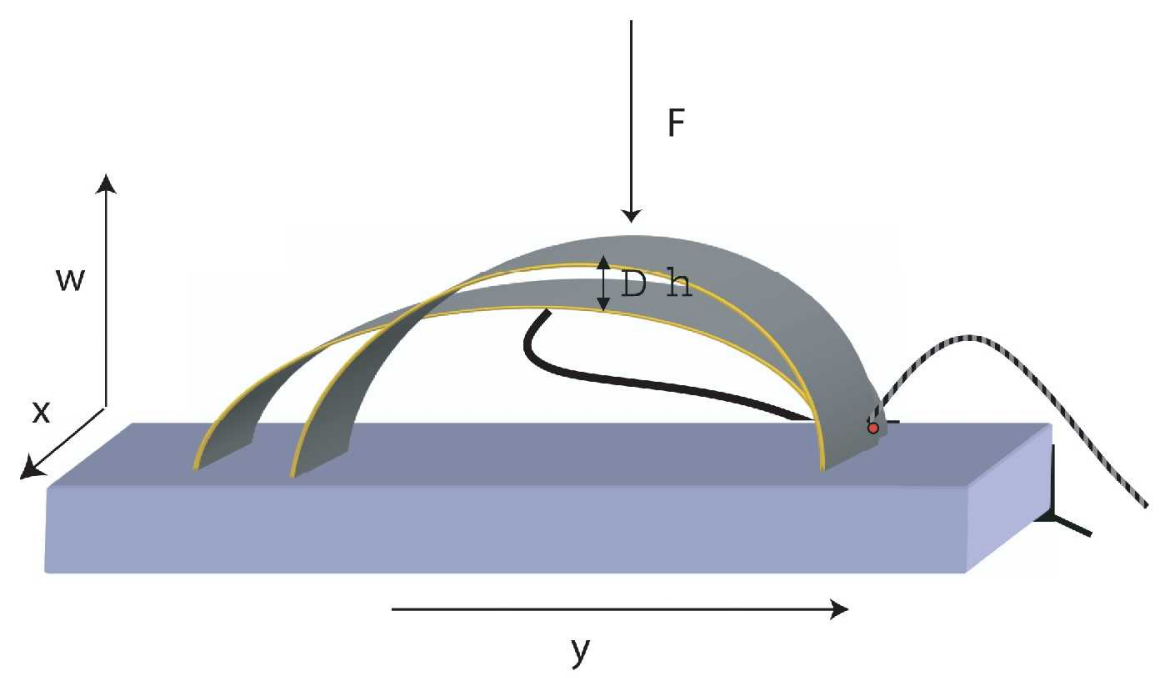

Figure 1. Experimental setup and Axis definitions.

As a simple example we take 3 layer actuator constructed from 1 mil brass, $1 \mathrm{mil}$ LaRC SI thermoplastic and 6.8 mil PZT 5A. By solving Equation 13 with $\mathrm{V}=0$ and plotting $\mathrm{F}$ as a function of dome height we find the spring force for the actuator. The results of this calculation show the spring force is multivalued. When initially in the short axis mode, the actuator may be depressed until a critical load is reached. At this point, the actuator switches and locks to the long axis solution. This has been verified experimentally and is a general characteristic of all Thunder actuators that have unequal $\mathrm{x}$ and $y$ curvatures. When the $\mathrm{x}$ and $\mathrm{y}$ curvatures are strongly unequal, the spring force is almost linear except for the bifurcation point. 


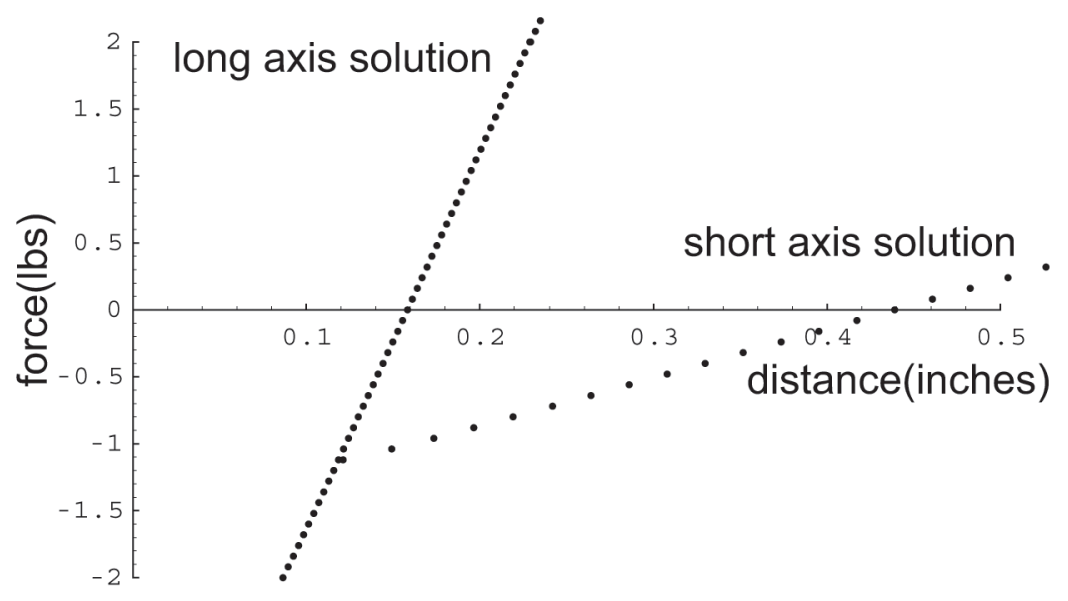

Figure 2. Calculation of spring force vs. distance for a 3-layer actuator.

\section{Charge and Voltage Generation}

If the device is used as a sensor a charge will be developed across the plates of the device. The magnitude of the charge will depend on the stress state of the device. The main contributions to this will be from the planar stress due to the mechanical advantage. The charge developed from a small piece of the device of dimensions $\mathrm{dx} d \mathrm{~d}$ is,

$$
d q=d_{31}\left(\sigma_{x}+\sigma_{y}\right)_{j} d x d y=d_{31}\left(Q_{1}+Q_{2}\right)_{j}\left(\varepsilon_{x}+\varepsilon_{y}\right) d x d y
$$

With this formulation the strains are functions of $\mathrm{x}$ and $\mathrm{y}$ and the PZT is assumed to be on the j'th layer. By Equation 10 we have, 


$$
\left(\varepsilon_{x}+\varepsilon_{y}\right)_{j}=c+d-\frac{1}{4} a b\left(x^{2}+y^{2}\right)-\frac{1}{2}(a+b)\left(z_{j-1}+z_{j}\right)
$$

where the strains we calculated are at the center of the PZT layer. The total charge generated is obtained by integrating over the surface. The result is,

$$
q=d_{31}\left(Q_{1}+Q_{2}\right)_{j}\left[c+d-\frac{1}{12} a b\left(L_{x}^{2}+L_{y}^{2}\right)-\frac{1}{2}(a+b)\left(z_{j-1}+z_{j}\right)\right] L_{x} L_{y}
$$

The voltage is obtained by dividing the total charge by the capacitance. The capacitance of the device is,

$$
C=\mu \frac{L_{x} L_{y}}{\mathrm{t}}
$$

where $\mu$ is the permittivity of PZT. The voltage is then,

$$
V=d_{31} \frac{\left(Q_{1}+Q_{2}\right)_{j} t}{\mu}\left[c+d-\frac{1}{12} a b\left(L_{x}^{2}+L_{y}^{2}\right)-\frac{1}{2}(a+b)\left(z_{j-1}+z_{j}\right)\right]
$$

To find the voltage one may solve Equation 13 for $a, b, c$ and $d$ in terms of the applied force and material properties. It should be noted we have ignored prestress effects on the planar charge constant, $d_{31}$. This charge constant is a function of stress as well.

\section{Voltage and power under load}


The equivalent circuit for a piezoelectric sensor for low frequencies is a voltage source in series with a capacitor. The magnitude of the voltage depends on how the material is stressed. For the case of Thunder, this voltage is given by Equation 20 . The capacitance is the capacitance of the device and is given by Equation 19.

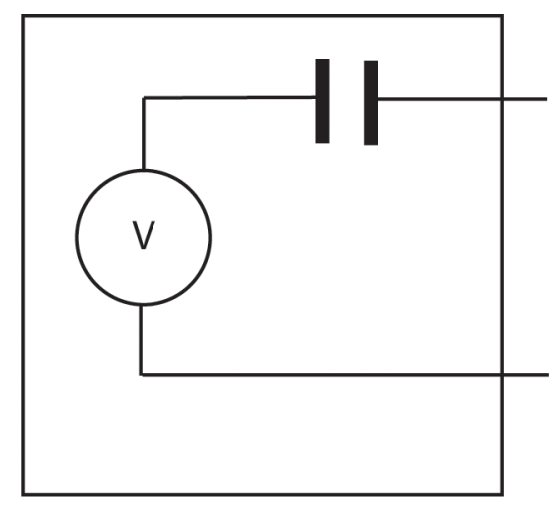

Figure 3. Equivalent circuit for a Thunder sensor (low frequency model).

To find the voltage and power under load we first add up all voltages around the loop for an external load, $R$. The result is,

$$
\frac{q}{C}+I R=\frac{q_{0}}{C}(1-\cos (\omega \mathrm{t}))
$$

Using $\mathrm{I}=\mathrm{dq} / \mathrm{dt}$ and rearranging Equation 21 we find,

$$
\frac{d q}{d t}+\frac{q}{R C}=\frac{q_{0}}{R C}(1-\cos (\omega t))
$$


The steady state solution (solution for long times) of this is,

$$
\begin{aligned}
& q=q_{0}+\frac{q_{0}}{\sqrt{1+C^{2} R^{2} \omega^{2}}} \sin (\omega t+\phi), \\
& \tan (\phi)=\frac{-1}{R C \omega}
\end{aligned}
$$

The current is then,

$$
I=\frac{d q}{d t}=\frac{\omega q_{0}}{\sqrt{1+C^{2} R^{2} \omega^{2}}} \cos (\omega t+\phi)
$$

The Power may be calculated from,

$$
P=I^{2} R=\frac{\omega^{2} q_{0}^{2} R}{1+C^{2} R^{2} \omega^{2}} \cos ^{2}(\omega t+\phi)
$$

The average power is,

$$
\langle P\rangle=\left\langle I^{2}\right\rangle R=\frac{1}{2} \frac{\omega^{2} q_{0}^{2} R}{1+C^{2} R^{2} \omega^{2}}
$$

By maximizing Equation 26 with respect to $R$, it can be shown that the power is maximized when,

$$
R=\frac{1}{C \omega}
$$


This is the point where there is a perfect impedance matching between the Thunder sensor and the electrical load, R.

\section{Experimental}

Voltage vs. displacement:

To compare this with actual manufactured THUNDERs, a piece measuring $3 "$ x $1.75^{\prime \prime}$ was selected and cycled in displacement from 0 to .3 inches at 1 to $10 \mathrm{~Hz}$. The device was constructed from 6.8 mil PZT5A, 1 mil LaRC SI and 2 mil brass, and had an initial dome height of approximately 0.7 inches. The material properties of the PZT were assumed to be $\mathrm{d} 31=-7.83 \times 10^{-10} \mathrm{C} / \mathrm{lb}$, Young's modulus of $\mathrm{Y}=9.1 \times 10^{6} \mathrm{psi}$, a $\mathrm{P}=.31$ Poisson's ratio, and a 1700 dielectric constant. The Brass assumed to be $\mathrm{Y}=1.5 \times 10^{7} \mathrm{psi}$ and $\mathrm{P}=.34$. The LaRC SI adhesive layer was assumed to be $\mathrm{Y}=5.8 \times 10^{5} \mathrm{psi}$ and $\mathrm{P}=.45$. The voltage output of the device was measured using an oscilloscope and recorded manually. Two samples are shown in the graphs. Figure 4 is a comparison between the theoretical curve and the measured data at $1 \mathrm{~Hz}$ and $10 \mathrm{~Hz}$. The theoretical curve was calculated based only on material properties, geometry and displacements. The $1 \mathrm{~Hz}$ curve starts to deviate from the ideal curve almost immediately whereas the $10 \mathrm{~Hz}$ curve matches more closely. The deviation in the $1 \mathrm{~Hz}$ curve could be due to creep in the PZT. Another possibility would be due to the impedance load of the measuring device, which unfortunately is not known due to the fact the data was collected by a summer student in 1997 who is now long gone. The THUNDER impedance is $1 /(\omega \mathrm{C})$, where $\mathrm{C}=2.87 \times 10^{-7}$ 
$\Omega$. With $Z=5.55 \times 10^{5} \Omega$ at $1 \mathrm{~Hz}$ and $Z=5.55 \times 10^{4} \Omega$ at $10 \mathrm{~Hz}$, a probe in the $1 \mathrm{M} \Omega-20 \mathrm{M} \Omega$ range, could easily cause the effects shown.

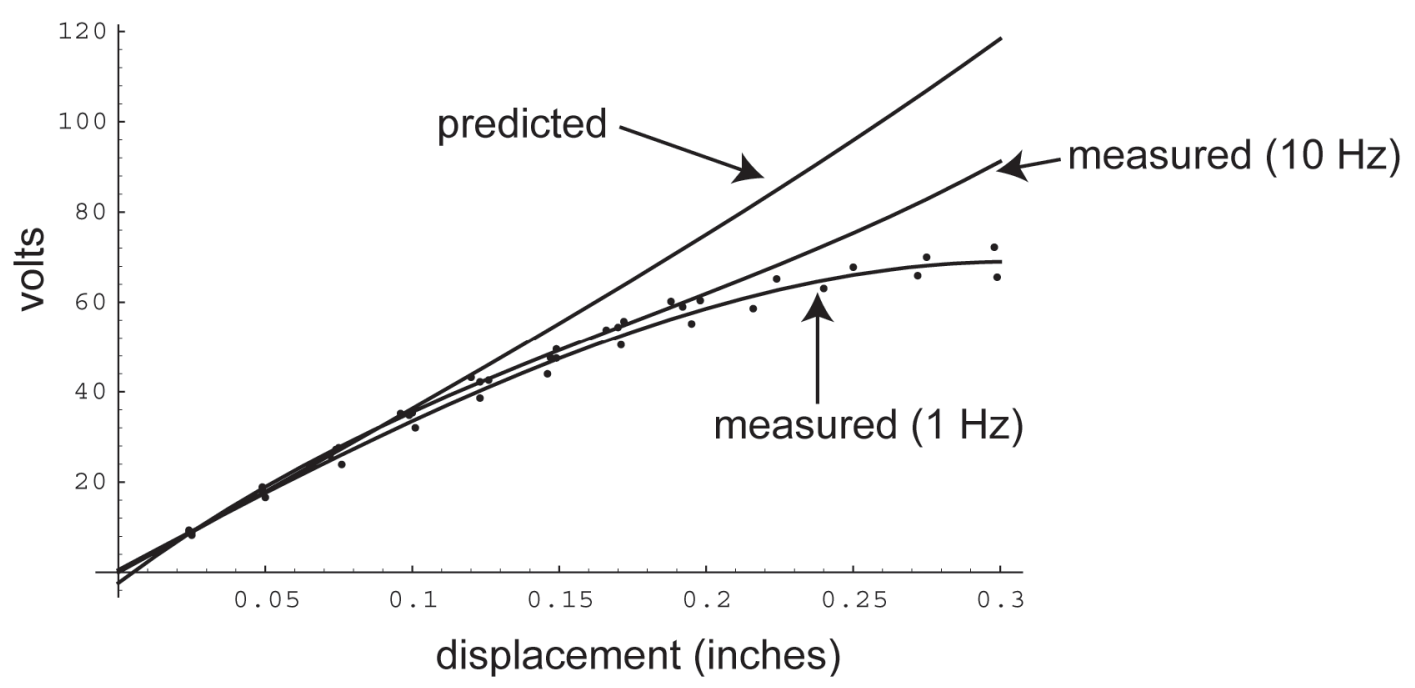

Figure 4. Voltage vs. displacement (two samples)

Voltage, power, and phase vs. electrical load:

To find power vs. electrical load the sample was cycled in displacement from 0 to .15 inches under various resistive loads. The voltage was measured across the resistor and the average power was calculated from $P=1 / 2 V^{2} / R$. This was then compared to the model based only on material properties, geometry and displacements. Here we see the deviation increases with increasing resistance. This could either be a material effect or possibly due to the impedance of the measuring device. In any even it shows the validity of Equation 26. The qualitative nature of the curves is virtually identical and the maximum power is obtained by matching the impedance of the sensor with the resistive 
load. The phase difference between the predicted and measured values indicates the impedance of the scope used to measure the voltage is significant.

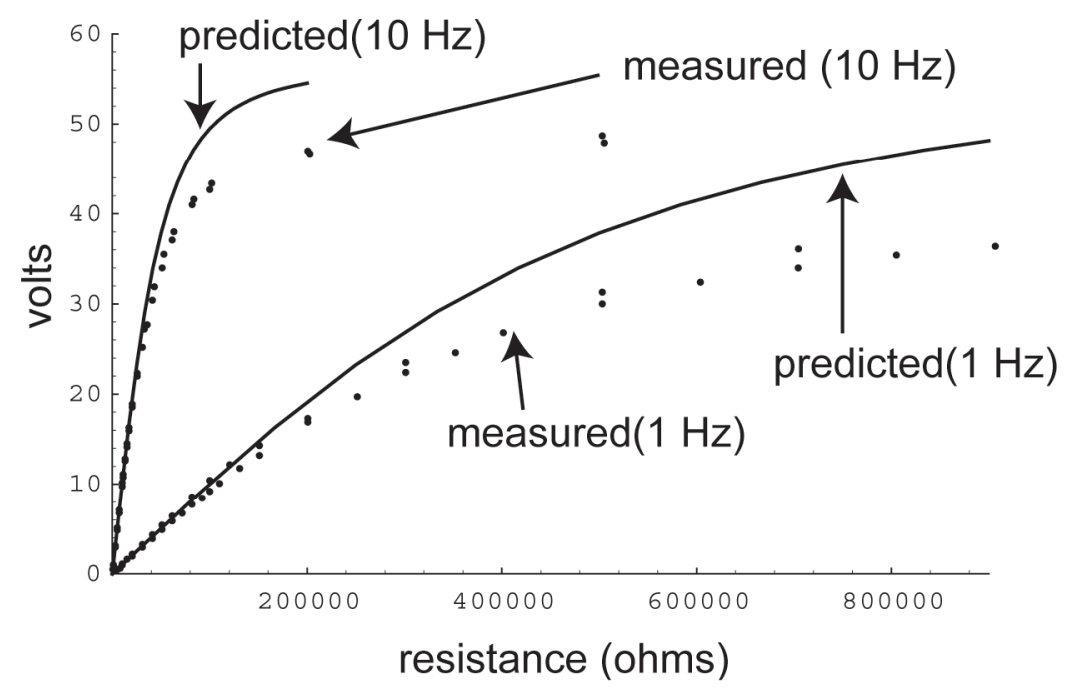

Figure 5. Voltage vs. resistance (two samples)

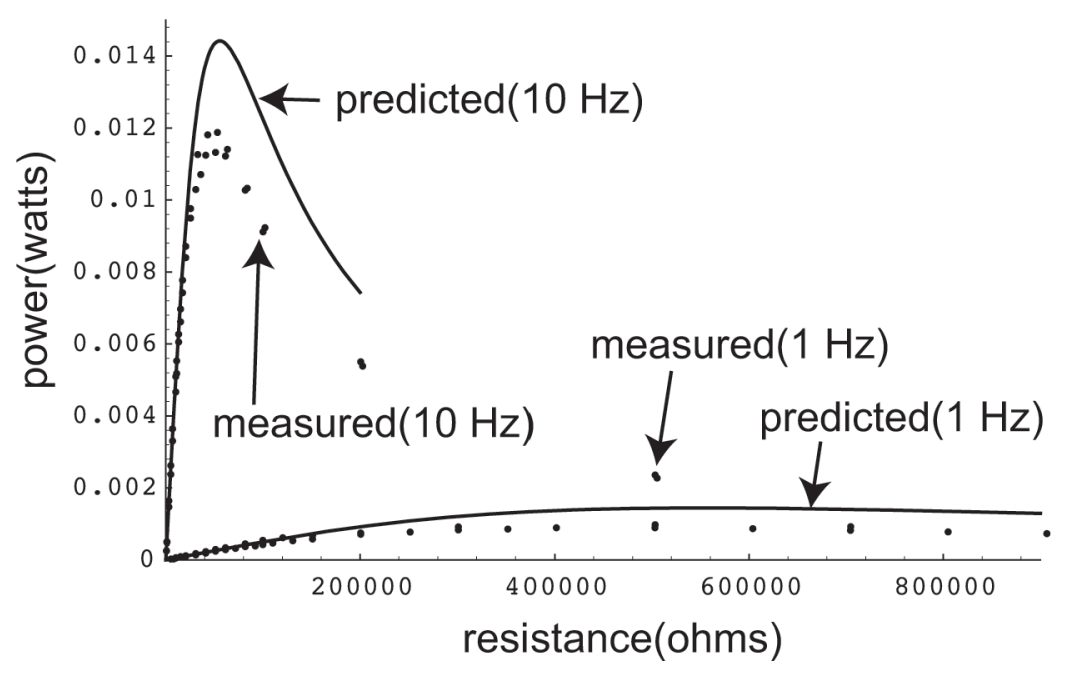

Figure 6. Power vs. resistance (two samples). 


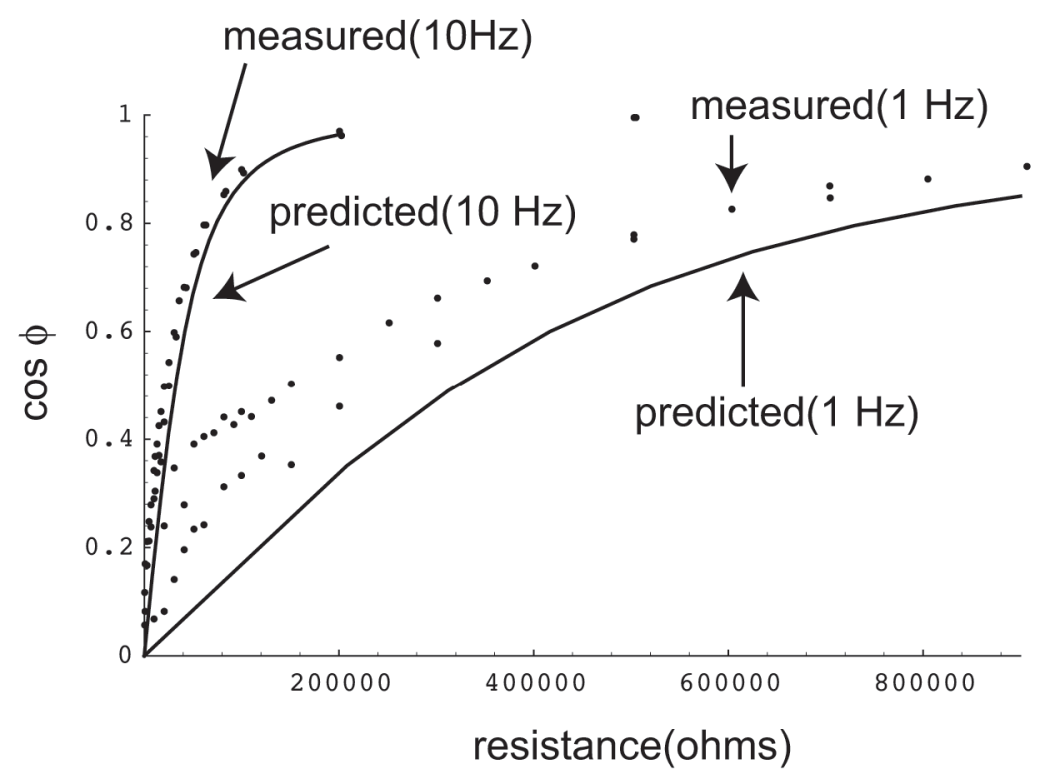

Figure 7. Relative phase vs. resistance (two samples).

\section{Conclusions}

These results show the basic assumptions used to predict the output of the devices are correct and it represents a very simple but useable model for low frequencies. It does, in fact, represent the first and only model not based on finite element analysis for the prediction of voltage outputs of THUNDER actuators as a function of displacement.

The impedance of the scope used to measure the voltage output is significant enough to affect the voltage output in situations where the load resistance was high, 
which one would expect. The error in that case was very small for low resistance and increased to about $20 \%$ over the range measured. A charge amplifier [21] would have been better to use in this type of situation since it eliminates the effects of the impedance of the scope. The maximum power measurements varied from the model by about 14$20 \%$ depending on the frequency. Although it is hard to see from the graphs, the $1 \mathrm{~Hz}$ case was slightly worse that the $10 \mathrm{~Hz}$ case. Likewise, the relative phase measurements for the $10 \mathrm{~Hz}$ case were better than the $1 \mathrm{~Hz}$ case as well.

More work needs to be done to both increase the sophistication of the model and to take further measurements using a charge amplifier or high impedance device to eliminate the effect of the impedance of the measuring device. 


\section{References}

1. Alan R.D. Curtis, A Model of the THUNDER Actuator

BBN Tech. Memo. No. 1190

NASA Contract NAS 1 20101, 12/31/97

http://hdl.handle.net/2060/20080042332

2. Taleghani, B. K. and Campbell, J. F., "Non-Linear Finite Element Modeling of THUNDER Piezoelectric Actuators," Proceedings of the SPIE Int. Soc. Opt., 3668, $555-566(1999)$

3. M. W. Hyer, Calculations of the Room-Temperature Shapes of Unsymmetric Laminates, J. Composite Materials

Vol. 15, July 1981

4. A. Hamamoto and M. W. Hyer Non-Linear Temperature-Curvature Relationships for Unsymmetric Graphite-Epoxy Laminates Int. J. Solid Structures

Vol 23, No.7,pp 919-935, 1987 
5. M. W. Hyer and P. C. Bhavani, Suppression of Anticlastic Curvature in Isotropic and Composite Plates, Int. J. Solid Structures

Vol. 20, No. 6, pp 553-570, 1987

6. Joel Campbell, Quasi-Static Analysis of LaRC THUNDER Actuators NASA patent disclosure, LAR-15827, April, 1998

Also NASA/TM-2007-214872

http://ntrs.nasa.gov/archive/nasa/casi.ntrs.nasa.gov/20070018753_2007019019.pdf

7. Joel Campbell, Blocked Force and Loading Calculations for LaRC THUNDER Actuators, NASA patent disclosure, LAR-15827, April, 1998

Also NASA/TM-2007-214875

http://ntrs.nasa.gov/archive/nasa/casi.ntrs.nasa.gov/20070018754_2007019020.pdf

8. Joel Campbell, Quasi-Static Analysis of Round LaRC THUNDER Actuators NASA patent disclosure, LAR-15827, April, 1998

Also NASA/TM-2007-214876

http://ntrs.nasa.gov/archive/nasa/casi.ntrs.nasa.gov/20070021226_2007019077.pdf

9. Hyer, M.W., and A Jilani, "Predicting the Deformation Characteristics of Rectangular Unsymmetrically Laminated Piezoelectric Materials," Smart Materials and Structures, 7, $784-791$ (1998). 
10. Hyer, Michael W. and Adel B. Jilani, Deformation characteristics of circular RAINBOW actuators, Smart Mater. Struct. 11 175-195, 2002

11. Hyer, Michael W. and Adel B. Jilani, "Manufactured Configurations of Piezoceramic Disk-Style Actuators," Journal of Intelligent Material Systems and Structures, 14, 359 -370 (2003)

12. Aimmanee, S., and Hyer, M. W., "Analysis of the Manufactured Shape of Rectangular THUNDER@-Type Actuators," Smart Materials and Structures, 13, $1389-1406$ (2004)

13. Mossi, K., Mouhli, M., Mane, P., Smith, B., and Bryant, R. (2006). "Shape modeling and validation of stressed biased piezoelectric actuators." Smart Materials and Structures, 15 (2006) 1785-1793.

14. Capozzoli, M., Gopalakrishnan, J., Hogan, K., Massad, J., Tokarchik, T., Wilmarth, S., Banks, H., Mossi, K., and Smith, R., "Modeling Aspects Concerning THUNDER Actuators," Proceedings of the SPIE Int. Soc. Opt., 3667, 719 - 727 (1999).

15. Ball, B.L., Smith, R.C. and Ounaies, Z., "A Dynamic Hysteresis Model for THUNDER@ Transducers," Proceedings of the SPIE Int. Soc Opt., 5049, 100 - 111 (2003). 
16. Lee, Sangki Byung C. Cho, Hoon C. Park, Nam S. Goo, and Kwang J. Yoon, "Piezoelectric Actuator-Sensor Analysis using a Three-Dimensional Assumed Strain Solid Element," Journal of Intelligent Material Systems and Structures, 15, 329- 338 (2004).

17. Park, Hoon C. Sangki Lee, Byung C. Cho, Kwang J. Yoon, Nam Seo Goo, “Threedimensional assumed strain solid element for piezoelectric actuator/sensor analysis," Proc. SPIE, Vol. 4326, p. 538 - 547, (2001).

18. S. G. Lekhnitskii

Anisotropic Plates

Gordon and Breach Publishers 1968

19. Libo Ren and Azar Parvizi Majidi, "A Model for the Shape Control of Cross-ply Laminated Shells using a Piezoelectric Actuator," Journal of Composite Materials, 40, 1271. 2006

20. R. M. Jones

Mechanics of Composite Materials

McGraw-Hill 1975

21. J. F. Campbell and D. S. Cairns, A Multi-Purpose Sensor for Composite Laminates Based on a Piezo-Electric Film, J. Composite Materials, Vol. 26, No.3 /1992 
